\title{
Modified homotopy perturbation method for solving hypersingular integral equations of the first kind
}

\author{
Z. K. Eshkuvatov ${ }^{1,3^{*}}$, F. S. Zulkarnain², N. M. A. Nik Long ${ }^{2}$ and Z. Muminov ${ }^{4}$
}

*Correspondence:
zainidin@usim.edu.my
${ }^{1}$ Faculty of Science
and Technology, Universiti
Sains Islam Malaysia (USIM),
Negeri Sembilan, Malaysia
Full list of author information
is available at the end of the
article

article

\begin{abstract}
Modified homotopy perturbation method (HPM) was used to solve the hypersingular integral equations (HSIEs) of the first kind on the interval $[-1,1]$ with the assumption that the kernel of the hypersingular integral is constant on the diagonal of the domain. Existence of inverse of hypersingular integral operator leads to the convergence of HPM in certain cases. Modified HPM and its norm convergence are obtained in Hilbert space. Comparisons between modified HPM, standard HPM, Bernstein polynomials approach Mandal and Bhattacharya (Appl Math Comput 190:1707-1716, 2007), Chebyshev expansion method Mahiub et al. (Int J Pure Appl Math 69(3):265-274, 2011) and reproducing kernel Chen and Zhou (Appl Math Lett 24:636-641, 2011) are made by solving five examples. Theoretical and practical examples revealed that the modified HPM dominates the standard HPM and others. Finally, it is found that the modified HPM is exact, if the solution of the problem is a product of weights and polynomial functions. For rational solution the absolute error decreases very fast by increasing the number of collocation points.
\end{abstract}

Keywords: Homotopy perturbation method, Hypersingular integral equation, Integral equation

Mathematics Subject Classification: 45E05 (Integral equations with kernels of Cauchy type ), 30 E20 (Integration, integrals of Cauchy type, integral representations of analytic functions)

\section{Background}

Hypersingular integral equations (HSIEs) arise a variety of mixed boundary value problems in mathematical physics such as water wave scattering (Kanoria and Mandal 2002), radiation problems involving thin submerged plates (Parsons and Martin 1994) and fracture mechanics (Chan et al. 2003; Nik Long and Eshkuvatov 2009). Chen and Zhou (2011) have solved HSIE using the improvement of reproducing kernel method. Golberg (1987) obtained the approximate solution of HSIEs using Galerkin and collacation method and discuss their convergence. Spline collocations method has also been used to solve linear HSIE of the first kind and nonlinear HSIE of the second kind in Boykov et al. (2010, 2014) respectively. Projection method with Chebyshev polynomials were 
discussed to solve the singular and hypersingular integral equation of the first kind in Eshkuvatov et al. (2009), Mahiub et al. (2011) respectively.

Homotopy perturbation method (HPM) has been used for a wide range of problems He (1999, 2000), Khan and Wu (2011), Madani et al. (2011), Ramos (2008), Słota (2010), Jafari et al. (2010), Golbabai and Javidi (2007), Dehghan and Shakeri (2008), Ghasemi et al. (2007), Panda et al. (2015), Okayama et al. (2011), Panda (2013), Javidi and Golbabai (2009), Ghorbani and Saberi-Nadjafi (2006), Mohamad Nor et al. (2013). Particularly, He $(1999,2000)$ was pioneer of establishing HPM and used it to solve the linear and nonlinear differential equations. Khan and Wu (2011) used He's polynomials to solve nonlinear problems. Madani et al. (2011) employed HPM together with Laplace transform for solving one-dimensional non-homogeneous partial differential equations with a variable coefficients. Other usage of HPM were finding the exact and approximate solutions of nonlinear ordinary differential equations (ODEs) (Ramos 2008), one-phase inverse Stefan problem (Słota 2010), linear and nonlinear integral equations (Jafari et al. 2010), the integro-differential equations (Golbabai and Javidi 2007; Dehghan and Shakeri 2008) and nonlinear Volterra-Fredholm integral equations Ghasemi et al. (2007). In Panda et al. (2015), a modified Lagrange approach is presented to obtain approximate numerical solutions of Fredholm integral equations of the second kind. The error bound is explained by the aid of several illustrative examples. In Okayama et al. (2011), two improved versions of the Sinc-collocation scheme are presented. The first version is obtained by improving the scheme so that it becomes more practical, and natural from a theoretical view point. In the second version, the variable transformation employed in the original scheme, the tanh transformation, is replaced with the double exponential transformation. It is proved that the replacement improves the convergence rate drastically. Numerical examples which support the theoretical results are also given. In Panda (2013), some recently developed analytical methods namely; homotopy analysis method, homotopy perturbation method and modified homotopy perturbation method are applied successfully for solving strongly nonlinear oscillators. The analytical results obtained by using HAM are compared with those of HPM, mHPM.

To improve the efficiency of the HPM, a few modifications have been made by many researches. For instance, Javidi and Golbabai (2009) added the accelerating parameter to the perturbation equation for obtaining the approximate solution for nonlinear Fredholm integral equation. Ghorbani and Saberi-Nadjafi (2006) added a series of parameter and selective functions to HPM to find the semi-analytical solutions of nonlinear Fredholm and Volterra integral equations. Mohamad Nor et al. (2013) developed the new homotopy function using De Casteljau algorithms to solve the algebraic nonlinear problems.

Consider HSIE of the first kind

$$
\frac{1}{\pi} f_{-1}^{1} \frac{K(x, t)}{(t-x)^{2}} \varphi(t) d t+\frac{1}{\pi} \int_{-1}^{1} L_{1}(x, t) \varphi(t) d t=f(x), \quad-1<x<1,
$$

where $\varphi(x)$ is the unknown function of $x$ to be determined, $K(s, t)$ and $L_{1}(s, t)$ are the square integrable kernels on $D=\left\{(s, t) \in \mathbb{R}^{2} \mid-1 \leq s, t \leq 1\right\}$. Assume that $K(s, t)$ is constant on the diagonal of the region, i.e.

$$
K(x, t)=c_{0}+(t-x) K_{1}(x, t),
$$


where $c_{0}$ is a nonzero constant and $K_{1}(x, t)$ is square integrable kernel of the form

$$
K_{1}(x, t)=Q(x)+(t-x) Q_{1}(x, t),
$$

$Q(x)$ is smooth function and $Q_{1}(x, t)$ is square integrable kernel.

The main objective is to find the bounded solution of Eq. (1). We search a solution in the form

$$
\varphi(x)=\sqrt{1-x^{2}} u(x) .
$$

Substituting Eqs. (2) and (3) into (1) yields

$$
\begin{aligned}
& \frac{c_{0}}{\pi} f_{-1}^{1} \frac{\sqrt{1-t^{2}}}{(t-x)^{2}} u(t) d t+\frac{Q(x)}{\pi} f_{-1}^{1} \frac{\sqrt{1-t^{2}}}{t-x} u(t) d t \\
& \quad+\frac{1}{\pi} \int_{-1}^{1} \sqrt{1-t^{2}} L(x, t) u(t) d t=f(x), \quad-1<x<1,
\end{aligned}
$$

where $L(x, t)=Q_{1}(x, t)+L_{1}(x, t)$.

Let us rewrite Eq. (4) in operator form

$$
H u+C u+L u=f,
$$

where

$$
\begin{aligned}
& H u(x)=\frac{c_{0}}{\pi} f_{-1}^{1} \frac{\sqrt{1-t^{2}}}{(x-t)^{2}} u(t) d t, \\
& C u(x)=\frac{Q(x)}{\pi} f_{-1}^{1} \frac{\sqrt{1-t^{2}}}{t-x} u(t) d t, \\
& L u(x)=\frac{1}{\pi} \int_{-1}^{1} \sqrt{1-t^{2}} L(x, t) u d t .
\end{aligned}
$$

In this paper, the standard (convex) HPM and the modification of improved HPM (in short modified HPM) are utilized to find the bounded approximate solution of HSIEs (4). Norm convergence for both HPM and modified HPM are proved.

The structure of this paper is arranged as follows. In "Hilbert spaces and operators" section, related information regarding to the Hilbert spaces and operators theory are given. Description of standard HPM and modified HPM are presented in "HPM and modified HPM for HSIEs" section. Norm convergence of both standard HPM and modified HPM are proved in "Convergence of the methods" section. Implementation of modified HPM and its comparisons with others are shown in "Numerical examples" section. Finally, "Conclusion" section is for the conclusion.

\section{Hilbert spaces and operators}

Let us consider some well known facts concerning the operator $H$ in Eq. (5). Let

$$
U_{n}(\theta)=\frac{\sin [(n+1) \theta]}{\sin \theta}, \quad \theta=\cos ^{-1} x, \quad n=0,1,2, \ldots,
$$

denote the Chebyshev polynomials of the second kind, and

$$
\phi_{n}=\sqrt{\frac{2}{\pi}} U_{n},
$$


is normalized Chebyshev polynomials of the second kind

$$
\int_{-1}^{1} \sqrt{1-t^{2}} \phi_{n}^{2}(t) d t=1
$$

It is well known that the hypersingular operator $H_{g}$ can be considered as the differential Cauchy operator i.e.,

$$
H_{g} u=\frac{1}{\pi} f_{-1}^{1} \frac{\sqrt{1-t^{2}} u(t)}{(t-x)^{2}} d t=\frac{d}{d x} \frac{1}{\pi} \int_{-1}^{1} \frac{\sqrt{1-t^{2}} u(t)}{t-x} d t=\frac{d}{d x} C_{g} u .
$$

as well as acting operator $C_{g}$ for Chebyshev polynomials of second kind yields

$$
C_{g} U_{n}(x)=\frac{1}{\pi} \int_{-1}^{1} \frac{\sqrt{1-t^{2}} U_{n}(t)}{t-x} d t=-T_{n+1}(x)
$$

where $T_{n+1}(x)$ is the Chebyshev polynomial of the first kind.

It can easily be shown from (7), (8), (9) and $T_{n+1}^{\prime}(x)=(n+1) U_{n}(x)$ that

$$
\begin{aligned}
& H \phi_{n}(x)=-c_{0}(n+1) \phi_{n}(x), \\
& C \phi_{n}(x)=-\frac{Q(x)}{2}\left(\phi_{n+1}(x)-\phi_{n-1}(x)\right), \quad n=0,1, \ldots,
\end{aligned}
$$

where $\phi_{-1}(x)=0$. Note that Eqs. (10) and (11) are crucial to the rest of our analysis.

Let $L(\rho)$ denotes the space of square integrable real valued function with respect to $\rho(x)=\sqrt{1-x^{2}}$. The inner product on $L(\rho)$ is given by

$$
\langle u, v\rangle_{\rho}=\int_{-1}^{1} \rho(t) u(t) v(t) d t,
$$

and $\|u\|_{\rho}=\sqrt{\langle u, v\rangle_{\rho}}$ denotes the norm.

The set $\left\{\phi_{k}\right\}_{k=0}^{\infty}$ is a complete orthonormal basis for $L(\rho)$, so that if $u \in L(\rho)$ then

$$
u=\sum_{k=0}^{\infty}\left\langle u, \phi_{k}\right\rangle_{\rho} \phi_{k},
$$

where the sum converges in $L(\rho)$. In addition, the norm of $u$ satisfies the Parseval's equality

$$
\|u\|_{\rho}^{2}=\sum_{k=0}^{\infty}\left\langle u, \phi_{k}\right\rangle_{\rho}^{2} .
$$

We will need the subspace of $L(\rho)$ which is consisting of all $u$ such that

$$
\sum_{k=0}^{\infty}(k+1)^{2}\left\langle u, \phi_{k}\right\rangle_{\rho}^{2}<\infty .
$$

All functions satisfying (12) is denoted by $L_{1}(\rho)$ and it can be made into Hilbert space if the inner product of $u \in L_{1}(\rho)$ and $v \in L_{1}(\rho)$ are defined by 


$$
\langle u, v\rangle_{1}=\sum_{k=0}^{\infty}(k+1)^{2}\left\langle u, \phi_{k}\right\rangle_{\rho}\left\langle v, \phi_{k}\right\rangle_{\rho} .
$$

The norm of $u \in L_{1}(\rho)$ is given by

$$
\|u\|_{1}^{2}=\sum_{k=0}^{\infty}(k+1)^{2}\left\langle u, \phi_{k}\right\rangle_{\rho}^{2}
$$

We extend the operator $H$ defined by (5) as a bounded operator from $L_{1}(\rho)$ to $L(\rho)$ by defining

$$
H u=c_{0} \sum_{k=0}^{\infty}\left\langle u, \phi_{k}\right\rangle_{\rho} H \phi_{k}=c_{0} \sum_{k=0}^{\infty}\left\langle u, \phi_{k}\right\rangle_{\rho}(-(k+1)) \phi_{k},
$$

and observe that

$$
\|H u\|_{\rho}^{2}=c_{0}^{2} \sum_{k=0}^{\infty}(k+1)^{2}\left\langle u, \phi_{k}\right\rangle_{\rho}^{2}=c_{0}^{2}\|u\|_{1}^{2} .
$$

It is not hard to show that $H^{-1}: L_{1}(\rho) \rightarrow L(\rho)$ exist and is given by

$$
H^{-1} u=\frac{1}{c_{0}} \sum_{k=0}^{\infty}\left(-\frac{\left\langle u, \phi_{k}>_{\rho}\right.}{k+1}\right) \phi_{k},
$$

hence $H$ is invertible Golberg (1987).

Lemma 1 The norm of operator $H^{-1}: L_{1}(\rho) \rightarrow L(\rho)$ is

$$
\left\|H^{-1}\right\|=\frac{1}{\left|c_{0}\right|}
$$

Proof Assume that $H^{-1} u=v$. On the other hand

$$
\left\langle v, \phi_{k}\right\rangle=\left\langle H^{-1} u, \phi_{k}\right\rangle=-\frac{1}{c_{0}} \frac{\left\langle u, \phi_{k}\right\rangle}{k+1} .
$$

Since $v \in L_{1}(\rho)$ and due to (16) we have

$$
\begin{aligned}
\|v\|_{1}^{2} & =\sum_{k=0}^{\infty}(k+1)^{2}\left(\left\langle v, \phi_{k}\right\rangle\right)^{2} \\
& =\frac{1}{c_{0}^{2}} \sum_{k=0}^{\infty}\left(\left\langle u, \phi_{k}\right\rangle\right)^{2} \\
& =\frac{1}{c_{0}^{2}}\|u\|_{\rho}^{2} .
\end{aligned}
$$


Therefore

$$
\left\|H^{-1} u\right\|_{1}=\frac{1}{\left|c_{0}\right|}\|u\|_{\rho}
$$

By the norm definition of operator, we obtain

$$
\left\|H^{-1}\right\|=\sup _{\substack{u \in L_{1}(\rho) \\\|u\|_{\rho} \leq 1}}\left\|H^{-1} u\right\|_{1}=\frac{1}{\left|c_{0}\right|} \sup _{\|u\|_{\rho} \leq 1}\|u\|_{\rho}=\frac{1}{\left|c_{0}\right|} .
$$

\section{Some facts from operators theory}

Lemma 2 Let $A, B$ be operators acting in Hilbert space. If $A$ is bounded and $B$ is compact then the products $A B$ and $B A$ are compact.

Lemma 2 is proven in Reed and Simon (1980, Theorem VI.12, pp. 200).

Lemma 3 The operators $C: L_{1}(\rho) \rightarrow L(\rho)$ and $H^{-1} C: L_{1}(\rho) \rightarrow L_{1}(\rho)$ are compact.

Proof Let us define operators $T_{r}, T_{l}: L_{1}(\rho) \rightarrow L(\rho)$ as

$$
\begin{aligned}
T_{r} u & =\sum_{k=0}^{\infty}\left\langle u, \phi_{k}\right\rangle_{\rho} \phi_{k+1}, \\
T_{l} u & =\sum_{k=1}^{\infty}\left\langle u, \phi_{k}\right\rangle_{\rho} \phi_{k-1} .
\end{aligned}
$$

These operators are bounded from $L(\rho) \rightarrow L(\rho)$. Moreover, boundedness and the compactly embeddability of $T_{r}$ and $T_{l}$ from $L_{1}(\rho)$ to $L(\rho)$ (Berthold Berthold et al. (1992, Conclusion 2.3)) implies the compactness of $T_{r}$ and $T_{l}$. From (11) and (18) it follows that

$$
C u(x)=\frac{Q(x)}{2}\left(T_{r}-T_{l}\right) u(x) .
$$

Since operators $T_{r}$ and $T_{l}$ are compact, its linear combinations is also compact i.e. $T_{r}-T_{l}: L_{1}(\rho) \rightarrow L(\rho)$. As we know $Q(x)$ is a continues function on the closed inter$\operatorname{val}[-1,1]$ and $T_{r}-T_{l}$ is compact, their product $C$ is also compact by Lemma 2 . On the other hand $H^{-1}$ is unitary and $C$ is compact due to Lemma 2. Hence, operator $H^{-1} C$ is compact.

Since operators $C$ and $L$ are compact then $C+L: L_{1}(\rho) \rightarrow L_{1}(\rho)$ is also compact. We know that $H^{-1}(C+L): L_{1}(\rho) \rightarrow L_{1}(\rho)$ is a compact operator. Due to the Fredholm theorem Reed and Simon (1980, Theorem VI.14) the inverse operator $\left(I+\lambda H^{-1}(C+L)\right)^{-1}$ of the operator function $I+\lambda H^{-1}(C+L), \lambda \in \mathbb{C}$, exists for all $\lambda$ in $\mathbb{C} \backslash C_{1}$, where $C_{1}$ is a discrete subset of $\mathbb{C}$ (i.e. a set $C_{1}$ has no limit points in $\mathbb{C}$ ) and for $\lambda \in C_{1}$ the null space $N\left(I+\lambda H^{-1}(C+L)\right)$ is finite, that is $z=-\lambda^{-1}$ is the eigenvalue of $H^{-1}(C+L)$ with finite multiplicity. These facts allows us to suppose the following 
Assumption $4 \lambda=1$ does not belong to $C_{1}$, i.e. $N\left(I+\lambda H^{-1}(C+L)\right)=\{0\}$.

Lemma 5 Let the Assumption 4 is satisfied, then the operator $H+C+L$ is invertible, and the main Eq. (5) has a unique solution.

Proof Since $H$ is invertible we get the relation

$$
H+C+L=H\left(I+\lambda H^{-1}(C+L)\right), \quad\left(I+\lambda H^{-1}(C+L)\right): L_{1}(\rho) \rightarrow L_{1}(\rho)
$$

which gives us the fact that $H+C+L$ is invertible iff $I+\lambda H^{-1}(C+L)$ is invertible. Then due to Assumption 4 the operator $H+C+L$ is invertible.

Assumption 6 Assume that $S=H+C+L$ where $H, C, L$ are defined by (5) is an invertible operator such that

$$
N(H+C+L)=0
$$

where $N(A)$ is a nullspace of $A$.

Let $P_{n}: L(\rho) \rightarrow L(\rho)$ be the orthogonal projection onto the subspace spaned by $\left\{\phi_{0}, \phi_{1}, \ldots, \phi_{n}\right\}$ and

$$
L_{n}=P_{n} L \text {. }
$$

Lemma 7 If (20) holds then

$$
\|\tilde{L}\|=\left\|L-L_{n}\right\|<\frac{\varepsilon}{\|\tilde{S}\|},
$$

and $\tilde{S}=\left(H+C+L_{n}\right)$ exist and invertible operator.

Proof Since $L$ is compact operator then $\tilde{L} \underset{n \rightarrow \infty}{\longrightarrow} 0$ i.e. for $\forall \varepsilon>0$, there exists $n_{0}$ such that $n \geq n_{0}$ implies

$$
\|\tilde{L}\|=\left\|L-L_{n}\right\|<\frac{\varepsilon}{\|\tilde{S}\|} .
$$

Due to invertibility of $S=H+C+L$ and $\tilde{L}=L-L_{n} \underset{n \rightarrow \infty}{\longrightarrow} 0$ we obtain

$$
\left\|(H+C+L)^{-1} \tilde{L}\right\| \leq 1 .
$$

Hence $I-(H+C+L)^{-1} \tilde{L}$ is invertible and

$$
\left(I-(H+C+L)^{-1} \tilde{L}\right)^{-1}(H+C+L)^{-1}=\left(H+C+L_{N}\right)^{-1}
$$

due to Lemma 2 operator $\tilde{S}=H+C+L_{n}$ is invertible

\section{HPM and modified HPM for HSIEs}

\section{HPM for HSIE}

We present the application of standard HPM for solving hypersingular integral equations of the first kind (5). The perturbation scheme in convex homotopy form is given by

$$
H^{*}(v, p)=(1-p)\left(H v-u_{0}\right)+p(H v+C v+L v-f),
$$


where $p \in[0,1]$ is homotopy parameter. For $p=0$ the solution of the operator equation $H^{*}(v, 0)=0$ is equivalent to the solution of a trivial problem $H v(x)-u_{0}(x)=0$. For $p=1$ the equation $H^{*}(v, 1)=0$ leads to the solution of Eq. (5).

The solution of operator equation $H^{*}(v, p)=0$ is searched in the form of power series

$$
v(x)=\sum_{k=0}^{\infty} p^{k} v_{k}(x)
$$

We assume that the series (22) possesses a radius of convergence not smaller than 1 . Substituting (22) into (5) yelds

$$
H\left(\sum_{k=0}^{\infty} p^{k} v_{k}(x)\right)=u_{0}+p\left[f-C\left(\sum_{k=0}^{\infty} p^{k} v_{k}(x)\right)-L\left(\sum_{k=0}^{\infty} p^{k} v_{k}(x)\right)-u_{0}\right] .
$$

Existence of $H^{-1}$ and equating the coefficients of like powers of $p$ in Eq. (23), leads to the following iterations

$$
\begin{aligned}
& v_{0}=H^{-1}\left(u_{0}\right), \\
& v_{1}=H^{-1}\left(f-u_{0}-C v_{0}-L v_{0}\right), \\
& v_{k}=H^{-1}\left(-C v_{k-1}-L v_{k-1}\right), \quad k \geq 2 .
\end{aligned}
$$

By computing the iterations $v_{k}$ in Eq. (24), we can find semi-analytical solution as follows

$$
\varphi(x)=\sqrt{1-x^{2}}\left(v_{0}(x)+v_{1}(x)+\cdots\right)
$$

Approximate solution can be computed by

$$
\begin{aligned}
\varphi_{N}(x) & =\sqrt{1-x^{2}}\left(v_{0}(x)+v_{1}(x)+\cdots+v_{N}(x)\right) \\
& =\sqrt{1-x^{2}} \tilde{v}_{N},
\end{aligned}
$$

where

$$
\tilde{v}_{N}=v_{0}(x)+v_{1}(x)+\cdots+v_{N}(x) .
$$

\section{Modified HPM for HSIEs}

Let us rewrite Eq. (5) in the equivalent form

$$
\tilde{S} u+\tilde{L} u=f,
$$

where

$$
\begin{aligned}
& S=H+C+L, \tilde{S}=H+C+L_{n} \text { with }\|S-\tilde{S}\| \underset{n \rightarrow \infty}{\longrightarrow} 0, \\
& \tilde{L}=L-L_{n}, \text { with }\|\tilde{L}\|=\left\|L-L_{n}\right\| \underset{n \rightarrow \infty}{\longrightarrow} 0 .
\end{aligned}
$$


Modified HPM for Eq. (28) is constructed as

$$
\begin{aligned}
H^{*}(v, p) & =(1-p)\left(\tilde{S} v-\sum_{j=0}^{m} \alpha_{j} g_{j}(x)\right)+p(\tilde{S} v+\tilde{L} v-f), \\
& =\tilde{S} v-\sum_{j=0}^{m} \alpha_{j} g_{j}(x)+p\left(\tilde{L} v+\sum_{j=0}^{m} \alpha_{j} g_{j}(x)-f\right) .
\end{aligned}
$$

Equating $H^{*}(v, p)=0$ leads to

$$
\tilde{S} v=\sum_{j=0}^{m} \alpha_{j} g_{j}(x)-p\left(\tilde{L} v+\sum_{j=0}^{m} \alpha_{j} g_{j}(x)-f\right) .
$$

Substituting series solution (22) into (29) yields

$$
\tilde{S}\left(\sum_{k=0}^{\infty} p^{k} v_{k}(x)\right)=\sum_{j=0}^{m} \alpha_{j} g_{j}(x)-p\left[\tilde{L}\left(\sum_{k=0}^{\infty} p^{k} v_{k}(x)\right)+\sum_{j=0}^{m} \alpha_{j} g_{j}(x)-f\right] .
$$

Equating both sides to the like power of $p$ gives

$$
\begin{aligned}
& \tilde{S}\left(v_{0}\right)=\sum_{j=0}^{m} \alpha_{j} g_{j}(x), \\
& \tilde{S}\left(v_{1}\right)=-\tilde{L}\left(v_{0}\right)+f-\sum_{j=0}^{m} \alpha_{j} g_{j}(x), \\
& \tilde{S}\left(v_{k}\right)=-\tilde{L}\left(v_{k-1}\right), \quad k=2,3, \ldots
\end{aligned}
$$

Since $\tilde{S}$ is invertible by Lemma 7, we have

$$
\begin{aligned}
& v_{0}=\tilde{S}^{-1}\left(\sum_{j=0}^{m} \alpha_{j} g_{j}(x)\right), \\
& v_{1}=\tilde{S}^{-1}\left(-\tilde{L}\left(v_{0}\right)+f-\sum_{j=0}^{m} \alpha_{j} g_{j}(x)\right), \\
& v_{k}=\tilde{S}^{-1}\left(-\tilde{L}\left(v_{k-1}\right)\right), \quad k=2,3, \ldots
\end{aligned}
$$

Semi-analytical solution of Eq. (5) can be computed by (25).

Remark Note that most cases of modified HPM, the unknown coefficients $\alpha_{j}$ of $v_{0}$ in the first equation of (31) are defined by equating the next iteration $v_{1}$ to be zero and it leads to $v_{k}=0, k \geq 2$ which implies two step method. In general, if $v_{1} \neq 0$ but $v_{1}^{(m)} \rightarrow 0$ as $m \rightarrow \infty$ then we can compute the next iteration $v_{k}, k \geq 2$. It effects to the next iteration $k \geq 2$ but the contribution to the solution of the problem will be very small therefore we can neglect it. 


\section{Convergence of the methods}

\section{Convergence of HPM}

Let us consider HSIE (5) by adding parameter $\lambda$ of the form

$$
H u+\lambda(C+L) u=f .
$$

Standard HPM for Eq. (32) has the scheme

$$
\begin{aligned}
& v_{0}=H^{-1}\left(u_{0}\right), \\
& v_{1}=H^{-1}\left(f-u_{0}-\lambda(C+L) v_{0}\right), \\
& v_{k}=H^{-1}\left(-\lambda(C+L) v_{k-1}\right), \quad k \geq 2 .
\end{aligned}
$$

Since $H^{-1}$ exists, Eq. (33) is computable. The convergence of the method is given in the following theorem.

Theorem 8 Let $K(x, t)=c_{0}+(t-x) K_{1}(x, t)$ and $K_{1}(x, t), L(s, t) \in C(D)$ and $f \in C[-1,1]$ be continuous functions. In addition, if the following inequality

$$
|\lambda|\|C+L\|<\left|c_{0}\right|
$$

holds and initial guess $u_{0}(t)$ is chosen as a continuous function for $t \in[-1,1]$, then the series (22) is norm convergent to the exact solution $u$ on the interval $[-1,1]$ for each $p=[0,1]$.

Proof Let $\left\|u_{0}\right\|_{\rho}=M$ and $\|f\|_{\rho}=M_{1}$. Based on (33) and Lemma 1 we have

$$
\begin{aligned}
\left\|v_{0}\right\|_{1} & \leq\left\|H^{-1}\right\|\left\|u_{0}\right\|_{\rho}=\frac{M}{\left|c_{0}\right|}, \\
\left\|v_{1}\right\|_{1} & \leq\left\|H^{-1}\right\|\left\|f-u_{0}-\lambda(C+L) v_{0}\right\|_{\rho}, \\
& \leq \frac{c_{0} M_{1}+c_{0} M+|\lambda|\|C+L\| M}{\left|c_{0}\right|^{2}}=B, \\
\left\|v_{2}\right\|_{1} & \leq\left\|H^{-1}\right\|\left\|\lambda(C+L) v_{1}\right\|_{\rho}, \\
& \leq \frac{|\lambda|\|C+L\| B}{\left|c_{0}\right|} \\
\left\|v_{k}\right\|_{1} & \leq\left(\frac{|\lambda|\|C+L\|}{\left|c_{0}\right|}\right)^{k-1} B, \\
& =\gamma_{1}^{k-1} B, \quad k \geq 2 .
\end{aligned}
$$

where

$$
\begin{aligned}
& \gamma_{1}=\frac{|\lambda|\|C+L\|}{\left|c_{0}\right|} \\
& B=\frac{1}{\left|c_{0}\right|^{2}}\left[c_{0} M_{1}+c_{0} M+|\lambda|\|C+L\| M\right] .
\end{aligned}
$$


Assume that $\gamma_{1}<1$, then from (22) at $p=1$, we obtain

$$
\begin{aligned}
\|v\|_{1} & \leq\left\|\sum_{k=0}^{\infty} v_{k}(x)\right\|_{1} \leq\left\|v_{0}\right\|_{1}+\sum_{k=1}^{\infty}\left\|v_{k}\right\|_{1}, \\
& \leq M+\sum_{k=1}^{\infty} \gamma_{1}^{k-1} B=M+\frac{B}{1-\gamma_{1}}<\infty .
\end{aligned}
$$

Therefore, series (22) converges to the exact solution in the sense of norm $\|\cdot\|_{1} \cdot \square$

Remark 9 Note that in our case $\lambda=1$ and the convergence of HPM can be established if and only if

$$
\|C+L\|<\left|c_{0}\right|
$$

It implies that HPM converges to the solution of HSIEs (5) in rare cases.

The first $N+1$ terms of series (22) as $p \rightarrow 1$ gives the approximate solution of the form

$$
\tilde{v}_{N}(x)=\sum_{k=0}^{N} v_{k}(x) .
$$

Theorem 10 If $\gamma_{1}<1$, then the rate of convergence of the approximate solution $\tilde{v}_{N}$ can be estimated by

$$
E_{N} \leq \frac{\gamma_{1}^{N}}{1-\gamma_{1}} B
$$

where $E_{N}=\left\|v(x)-\tilde{v}_{N}(x)\right\|_{1}$ and $B$ is defined by (36).

Proof

$$
\begin{aligned}
\left\|v(x)-\tilde{v}_{N}(x)\right\|_{1} & =\left\|\sum_{k=0}^{\infty} v_{k}(x)-\sum_{k=0}^{n} v_{k}(x)\right\|_{1} \\
& =\left\|\sum_{k=N+1}^{\infty} v_{k}(x)\right\|_{1} \leq \sum_{k=N+1}^{\infty}\left\|v_{k}(x)\right\|_{1} \\
& \leq \sum_{k=N+1}^{\infty} \gamma_{1}^{k-1} B \\
& =\frac{\gamma_{1}^{N}}{1-\gamma_{1}} B .
\end{aligned}
$$

Since $\gamma_{1}<1$, the norm

$$
\left\|v(x)-v_{n}(x)\right\|_{1} \rightarrow 0
$$

whenever $n \rightarrow \infty$. 


\section{Convergence of modified HPM}

Let us consider HSIE in the form of Eq. (28). If $v_{1}=0$ in (30), then $v_{0}$ satisfies $\tilde{S} v_{0}+\tilde{L} v_{0}=f$ and coincides with the exact solution. If $v_{1} \neq 0$ then $v_{1}^{(m)} \rightarrow 0$ i.e. for any $\varepsilon$, there exists $m_{0}$, such that $m>m_{0}$ implies

$$
\left\|v_{1}^{(m)}\right\|_{1}<\varepsilon
$$

Let $\left.\| \sum_{j=0}^{m} \alpha_{j} g_{j}(x)\right) \|_{\rho}=M_{2}$ and $\|f\|_{\rho}=M_{1}$, then due to (31) and existence of $S^{-1}$ (Lemma 6) we obtain

$$
\begin{aligned}
\left\|v_{0}\right\|_{1}= & \left\|\tilde{S}^{-1}\left(\sum_{j=0}^{m} \alpha_{j} g_{j}(x)\right)\right\|_{1} \\
& \leq\left\|\tilde{S}^{-1}\right\| M_{2}, \\
\left\|\nu_{1}\right\|_{1}= & \left\|\tilde{S}^{-1}\left(-\tilde{L}\left(v_{0}\right)-\sum_{j=0}^{m} \alpha_{j} g_{j}(x)+f\right)\right\|_{1} \\
& \leq \varepsilon, \\
\left\|v_{2}\right\|_{1}= & \left\|\tilde{S}^{-1}\left(\tilde{L}\left(v_{1}\right)\right)\right\|_{1} \\
& \leq\left\|\tilde{S}^{-1}\right\|\|\tilde{L}\|\left\|v_{1}\right\|_{1} \\
& \leq \gamma_{2} \varepsilon,
\end{aligned}
$$

where $\gamma_{2}=\left\|\tilde{S}^{-1}\right\|\|\tilde{L}\|$ and by continuing these procedure

$$
\begin{aligned}
\left\|v_{k}\right\|_{1}= & \left\|\tilde{S}^{-1}\left(\tilde{L}\left(v_{k-1}\right)\right)\right\|_{1} \\
& \leq\left\|\tilde{S}^{-1}\right\|^{k-1}\|\tilde{L}\|^{k-1}\left\|v_{1}\right\|_{1} \\
= & \gamma_{2}^{k-1} \varepsilon .
\end{aligned}
$$

Due to $\|\tilde{L}\|=\left\|L-L_{n}\right\| \underset{n \rightarrow \infty}{\longrightarrow} 0$ it can be easily shown that $\gamma_{2}<1$ for large enough $k$ then

$$
\begin{aligned}
\|v\|_{1} \leq & \left\|\sum_{k=0}^{\infty} v_{k}\right\|_{1}=\left\|v_{0}\right\|_{1}+\left\|\sum_{k=1}^{\infty} v_{k}\right\|_{1} \\
& \leq\left\|\tilde{S}^{-1}\right\| M_{2}+\sum_{k=1}^{\infty} \gamma_{2}^{k-1} \varepsilon \\
& =\left\|\tilde{S}^{-1}\right\| M_{1}+\frac{\varepsilon}{1-\gamma_{2}}<\infty .
\end{aligned}
$$

Thus, we have proved the following theorem

Theorem 11 Let $K(x, t)=c_{0}+(t-x) K_{1}(x, t)$ and $K_{1}(x, t), L(s, t) \in C(D)$ and $f \in C[-1,1]$ be continuous functions. In addition, if the following inequality

$$
\gamma_{2}=\left\|\tilde{S}^{-1}\right\|\|\tilde{L}\|<1
$$


holds and selective functions $g_{j}(x), j=0, \ldots, N$ are chosen as a continuous function on the interval $[-1,1]$, then the series solution (22) is norm convergent to the exact solution $\varphi(x)$ on the interval $[-1,1]$ for each $p=[0,1]$.

Remark 12 Theorems 8 and 11 show the fact that the exact solution $u$ belongs to $L_{1}(\rho)$. Then due to Berthold et al. (1992, Theorem 2.13) the function $u$ belongs to $C^{(1)}(-1,1)$.

Approximate solution of Eq. (4) in series (37) can be estimated as follows.

Theorem 13 Rate of convergence of approximate solution $\tilde{v}_{N}$ can be estimated by

$$
E_{N} \leq \frac{\gamma_{2}^{N}}{1-\gamma_{2}} \varepsilon
$$

where $E_{n}=\left\|v(x)-\tilde{v}_{N}(x)\right\|$ and $\varepsilon$ are defined by (39) and $\gamma_{2}<1$.

Proof

$$
\begin{aligned}
\left\|v(x)-\tilde{v}_{N}(x)\right\|_{1} & =\left\|\sum_{k=0}^{\infty} v_{k}(x)-\sum_{k=0}^{N} v_{k}(x)\right\|_{1} \\
& =\left\|\sum_{k=N+1}^{\infty} v_{k}(x)\right\|_{1} \leq \sum_{k=N+1}^{\infty}\left\|v_{k}(x)\right\|_{1} \\
& \leq \sum_{k=N+1}^{\infty} \gamma_{2}^{k-1} \varepsilon \\
& =\frac{\gamma_{2}^{N}}{1-\gamma_{2}} \varepsilon .
\end{aligned}
$$

Remark 14 Since $\gamma_{2}<1$, the term $\frac{\gamma_{2}^{N}}{1-\gamma_{2}} \varepsilon \rightarrow 0$ as $N \rightarrow \infty$. Moreover, sufficiently small $\varepsilon$ gives the smaller error rate for $E_{N}$ in (43) than error $E_{N}$ in (38). This fact shows that the modified HPM is dominates the standard HPM.

\section{Numerical examples}

Example 1 (Mandal and Bhattacharya 2007). Consider HSIE (1) of the form

$$
\frac{1}{\pi} f_{-1}^{1} \frac{\varphi(t)}{(x-t)^{2}} d t=1, \quad-1<x<1
$$

The exact solution of Eq. (44) is $\varphi(x)=-\sqrt{1-x^{2}}$ and $c_{0}=1, f(x)=1$.

Solution It is easy to find that Eq. (44) satisfied all conditions in Theorem 8. To apply HPM, we choose the initial guess as $u_{0}=\phi_{0}(x)$. Since $C u=L u \equiv 0$ and from (14) we can easily get 


$$
H^{-1}\left(\phi_{j}\right)=-\frac{1}{c_{0}} \frac{\phi_{j}}{j+1}
$$

Referring to (24) and using (45), we obtain successive functions

$$
\begin{aligned}
& v_{0}(x)=H^{-1}\left(\phi_{0}\right)=-\phi_{0}, \\
& v_{1}(x)=H^{-1}\left(1-\phi_{0}\right)=\left(-1+\phi_{0}\right), \\
& v_{j}(x)=0 . j=2,3, \ldots
\end{aligned}
$$

Since $v_{2}(x)=v_{3}(x)=\cdots=0$, the approximate solution of Eq. (44) is

$$
\varphi(x)=\sqrt{1-x^{2}}\left(v_{0}(x)+v_{1}(x)\right)=-\sqrt{1-x^{2}},
$$

which is identical with exact solution.

For application of modified HPM to the Eq. (44), we do this following steps:

1. Let selective functions $g_{j}(x)=\phi_{j}(x), j=0, \ldots m$. Since $\tilde{S}=H, \tilde{L} \equiv 0$ for (44) we can use inverse operator (45). Based on the scheme (31) for $m=2$ we obtain,

$$
\begin{aligned}
v_{0}(x) & =H^{-1}\left(\alpha_{0} \phi_{0}+\alpha_{1} \phi_{1}+\alpha_{2} \phi_{2}\right)=-\alpha_{0} \phi_{0}-\frac{\alpha_{1}}{2} \phi_{1}-\frac{\alpha_{2}}{3} \phi_{2}, \\
v_{1}(x) & =H^{-1}\left(1-\alpha_{0} \phi_{0}-\alpha_{1} \phi_{1}-\alpha_{2} \phi_{2}\right)=-1+\alpha_{0} \phi_{0}+\frac{\alpha_{1}}{2} \phi_{1}+\frac{\alpha_{2}}{3} \phi_{2}, \\
v_{k} & =H^{-1}(0) \equiv 0, k=2,3, \ldots
\end{aligned}
$$

2. Since $v_{k} \equiv 0, k=2,3, \ldots$ we can easily find approximate solution as

$$
\varphi(x)=\sqrt{1-x^{2}}\left(v_{0}(x)+v_{1}(x)\right)=-\sqrt{1-x^{2}},
$$

which coincides with exact solution.

Mandal and Bhattacharya (2007) consider the Eq. (44) and comparisons with HPM, modified HPM are summarized in Table 1.

Example 2 Mahiub et al. (2011). Consider HSIE of the form

$\frac{1}{\pi} \int_{-1}^{1} \frac{\varphi(t)}{(x-t)^{2}} d t+\frac{1}{\pi} \int_{-1}^{1} \sin (x) t^{4} \varphi(t) d t=-5\left(16 x^{4}-12 x^{2}+1\right)-\frac{\sin (x)}{32}, \quad-1<x<1$,

with exact solution $\varphi(x)=\sqrt{1-x^{2}}\left(16 x^{4}-12 x^{2}+1\right)$.

Table 1 Errors of methods for Eq. (44)

\begin{tabular}{llll}
\hline $\boldsymbol{x}$ & Error in Mandal and Bhattacharya (2007) & Error of HPM & Error of modified HPM \\
\hline-1 & 0 & 0 & 0 \\
-0.5 & $1.3 \times 10^{-17}$ & 0 & 0 \\
0 & 0 & 0 & 0 \\
0.5 & $7.8 \times 10^{-18}$ & 0 & 0 \\
1 & 0 & 0 & 0 \\
\hline
\end{tabular}


Solution Conditions of the Theorem 8 does not hold for Example 2. Therefore we did comparisons between modified HPM and method given in Mahiub et al. (2011).

To solve the Eq. (48) by modified HPM we do the following steps:

1. Let us choose selective functions $g_{j}(x)=\phi_{j}(x), j=0, \ldots, m$ and kernel $L(x, t)=\sin (x) t^{4}$ in Eq. (48) be approximated by projection kernel $L_{n}(x, t)=\sum_{i=1}^{l} b_{i}(x) \phi_{i}(t)$. In this case $\tilde{L} u=L u-L_{n} u \equiv 0$. Since $C u \equiv 0, \tilde{L} u \equiv 0$ then $\tilde{S}=H+L$. From (30) it follows that

$$
\begin{aligned}
& (H+L) v_{0}=\sum_{j=0}^{m} \alpha_{j} \phi_{j}(x), \\
& (H+L) v_{1}=f-\sum_{j=0}^{m} \alpha_{j} \phi_{j}(x) . \\
& (H+L) v_{k}=-\tilde{L}\left(v_{k-1}\right) \equiv 0, \quad k=2,3, \ldots
\end{aligned}
$$

2. Let $v_{o}=u_{0}=\sum_{j=0}^{m} \alpha_{j} \phi_{j}(x)$, then from the first equation of (49) we define

$$
H+L=I \text {. }
$$

From the 2nd equation of (49) we obtain

$$
\sum_{j=0}^{m} \alpha_{j} \phi_{j}(x)=f(x)
$$

Approximating $\sin (x)$ by Chebyshev polynomials

$$
\sin (x) \approx \sqrt{\frac{\pi}{2}}\left(\frac{11}{24} \phi_{1}(x)-\frac{1}{48} \phi_{3}(x)\right)
$$

and using first equation of (49) and taking account of (51) we get

$$
(H+L) \sum_{j=0}^{m} \alpha_{j} \phi_{j}(x)=\sqrt{\frac{\pi}{2}}\left(-5 \phi_{4}(x)-\frac{11}{24} \phi_{1}(x)+\frac{1}{48} \phi_{3}(x)\right) .
$$

Comparing the base of Chebyshev polynomial from the both sides of Eq. (53) the solutions are

$$
\alpha_{4}=\sqrt{\frac{\pi}{2}}, \quad \alpha_{1}=\alpha_{2}=\alpha_{3}=0 .
$$

3. Substituting (54) into Eq. (25) yields the exact solution

$$
\varphi(x)=\sqrt{1-x^{2}}\left(16 x^{4}-12 x^{2}+1\right) .
$$

Comparisons of Modified HPM and Chebyshev expansion Mahiub et al. (2011) is given in Table 2 for Eq. (48).

Example 3 Chen and Zhou (2011). Consider HSIE in the form

$$
\frac{1}{\pi} f_{-1}^{1} \frac{\varphi(t)}{(x-t)^{2}} d t+\frac{1}{2 \pi} \int_{-1}^{1} t x \varphi(t) d t=-8 x^{3}+\frac{17}{8} x-1, \quad-1<x<1,
$$


Table 2 Errors of approximate solutions for Eq. (48)

\begin{tabular}{lll}
\hline $\boldsymbol{x}$ & Mahiub et al. (2011) & Modified HPM \\
\hline-1 & 0 & 0 \\
-0.8 & $2.1 \times 10^{-10}$ & 0 \\
-0.6 & $4.0 \times 10^{-9}$ & 0 \\
-0.4 & $3.4 \times 10^{-9}$ & 0 \\
-0.2 & $2.3 \times 10^{-9}$ & 0 \\
0 & $2.0 \times 10^{-9}$ & 0 \\
0.2 & $2.3 \times 10^{-9}$ & 0 \\
0.4 & $3.3 \times 10^{-9}$ & 0 \\
0.6 & $4.0 \times 10^{-9}$ & 0 \\
0.8 & $2.7 \times 10^{-9}$ & 0 \\
1 & 0 & 0 \\
\hline
\end{tabular}

with exact solution $\varphi(x)=\sqrt{1-x^{2}}\left(1+2 x^{3}\right)$.

Conditions of Theorem 8 are satisfied, therefore for HPM we choose initial guess as $u_{0}=\phi_{1}(x)$. Errors of numerical solution, computed for $N=\{5,10\}$ where $N$ is a number of iteration, are given in Table 3.

To use modified HPM for solving Eq. (56), we do the following steps:

1. As usual we choose selective functions as $g_{j}(x)=\phi_{j}(x), j=0, \ldots, m$ and kernel $L(x, t)=t x$ in Eq. (56) be approximated by projection kernel of the form $L_{n}(x, t)=\sum_{i=1}^{l} b_{i}(x) \phi_{i}(t)$. Again for this case $\tilde{L} u=L u-L_{n} u \equiv 0$. Since $C u \equiv 0, \tilde{L} u \equiv 0$ then $\tilde{S}=H+L$. Using (30) we have

$$
\begin{aligned}
& (H+L) v_{0}=\sum_{j=0}^{m} \alpha_{j} \phi_{j}(x), \\
& (H+L) v_{1}=f-\sum_{j=0}^{m} \alpha_{j} \phi_{j}(x) . \\
& (H+L) v_{k}=-\tilde{L}\left(v_{k-1}\right) \equiv 0, k=2,3, \ldots
\end{aligned}
$$

2. Again $v_{k} \equiv 0, \quad k=2,3, \ldots$ and by equating $v_{1}=0$ we have

$$
\sum_{j=0}^{m} \alpha_{j} \phi_{j}(x)=f(x) .
$$

\section{Table 3 Errors of solutions for Eq. (56) solved by HPM}

\begin{tabular}{lll}
\hline $\boldsymbol{x}$ & $\boldsymbol{N}=\mathbf{5}$ & $\boldsymbol{N}=\mathbf{1 0}$ \\
\hline-0.9999 & $2.424502288 \times 10^{-8}$ & $2.312185561 \times 10^{-14}$ \\
-0.901 & $6.701859769 \times 10^{-7}$ & $6.391391532 \times 10^{-13}$ \\
-0.725 & $8.561715506 \times 10^{-7}$ & $8.165088181 \times 10^{-13}$ \\
-0.436 & $6.727677283 \times 10^{-7}$ & $6.416013033 \times 10^{-13}$ \\
-0.015 & $2.571605139 \times 10^{-8}$ & $2.452473774 \times 10^{-14}$ \\
0.015 & $2.571605139 \times 10^{-8}$ & $2.452473774 \times 10^{-14}$ \\
0.436 & $6.727677283 \times 10^{-7}$ & $6.416013033 \times 10^{-13}$ \\
0.725 & $8.561715506 \times 10^{-7}$ & $8.165088181 \times 10^{-13}$ \\
0.901 & $6.701859769 \times 10^{-7}$ & $6.391391532 \times 10^{-13}$ \\
0.9999 & $2.424502288 \times 10^{-8}$ & $2.312185561 \times 10^{-14}$ \\
\hline
\end{tabular}


using 1st equation of (57) and taking into account (58) yields

$(H+L) \sum_{j=0}^{m} \alpha_{j} \phi_{j}(x)=\sqrt{\frac{\pi}{2}}\left(-\phi_{3}(x)-\frac{15}{16} \phi_{1}(x)-\phi_{0}(x)\right)$.

Comparing the base of Chebyshev polynomial from the both sides of Eq. (59) produce a system. Solutions of the system are

$$
\alpha_{0}=\sqrt{\frac{\pi}{2}}, \quad \alpha_{1}=\frac{1}{2} \sqrt{\frac{\pi}{2}}, \quad \alpha_{2}=0, \quad \alpha_{3}=\frac{1}{4} \sqrt{\frac{\pi}{2}} .
$$

3. Substituting (60) into Eq. (25) yields

$$
\varphi(x)=\sqrt{1-x^{2}}\left(1+2 x^{3}\right) .
$$

which is identical with the exact solution.

Results are calculated by taking the maximum of absolute errors for Eq. (56). Comparison of the results between HPM, modified HPM and reproducing kernel Chen and Zhou (2011) shown in Table 4.

Example 4 Solve HSIE of the form

$$
\begin{aligned}
& \frac{1}{\pi} f_{-1}^{1} \frac{1+2(t-x)}{(x-t)^{2}} \varphi(t) d t+\frac{1}{2 \pi} \int_{-1}^{1} e^{2 x} t^{3} \varphi(t) d t=-16 x^{4}-40 x^{3}+4 x^{2} \\
& \quad+22 x+1+\frac{1}{32} e^{2 x}, \quad-1<x<1 .
\end{aligned}
$$

The exact solution of Eq. (62) is $\varphi=\sqrt{1-x^{2}}\left(8 x^{3}+4 x^{3}-4 x-1\right)$.

Solution For this example, the conditions of Theorem 8 does not hold. Therefore, HPM is not a reliable method to solve Eq. (62).

To obtain the approximate solutions of Eq. (62) by modified HPM (30), we do the following steps:

1. Approximate $L(x, t)=\frac{e^{2 x} t^{3}}{2}$ by Chebyshev polynomials

$$
L_{n}(x, t)=\frac{e^{2 x}}{16}\left(\phi_{3}(t)-2 \phi_{1}(t)\right)=L(x, t),
$$

therefore $\tilde{L}_{n} \equiv 0$. Choose selective functions $g_{j}(x)=\phi_{j}(x)$, then from (30), we have

$$
(H+C) v_{0}=\sum_{j=0}^{m} \alpha_{j} \phi_{j}(x)
$$

Table 4 Errors of solutions for Eq. (44)

\begin{tabular}{llll}
\hline $\boldsymbol{N}$ & Chen and Zhou (2011) & HPM & Modified HPM \\
\hline 5 & $6.8 \times 10^{-6}$ & $8.57 \times 10^{-7}$ & 0 \\
10 & $5.2 \times 10^{-8}$ & $7.8 \times 10^{-13}$ & 0 \\
\hline
\end{tabular}


$(H+C) \nu_{1}=f-\sum_{j=0}^{m} \alpha_{j} \phi_{j}(x)$.

$(H+C) v_{k}=-\tilde{L}\left(v_{k-1}\right) \equiv 0$,

with $v_{0}=\sum_{j=0}^{m} b_{j} \phi_{j}(x)$.

2. Since $v_{k} \equiv 0$, for $k \geq 2$ and approximating $e^{2 x}$ into Chebyshev polynomials with 4 bases

$e^{2 x} \simeq \sqrt{\frac{\pi}{2}}\left(\frac{19}{12} \phi_{0}(x)+\frac{4}{3} \phi_{1}(x)+\frac{5}{8} \phi_{2}(x)+\frac{1}{6} \phi_{3}(x)-\frac{1}{24} \phi_{4}(x)\right)$,

then substituting (67) into (65) and equating $v_{1}=0$ for $m=4$ yields

$$
\begin{gathered}
b_{0}=\frac{19}{384} \sqrt{\frac{\pi}{2}}, \quad b_{1}=\frac{25}{24} \sqrt{\frac{\pi}{2}}, \quad b_{2}=-\frac{507}{256} \sqrt{\frac{\pi}{2}}, \quad b_{3}=-\frac{959}{192} \sqrt{\frac{\pi}{2}}, \\
b_{4}=-\frac{767}{768} \sqrt{\frac{\pi}{2}} .
\end{gathered}
$$

3. From (64), we obtain the values of $\alpha_{k}, k=0,1, \ldots, 4$.

$$
\alpha_{0}=\alpha_{1}=\alpha_{4}=0, \quad \alpha_{2}=\alpha_{3}=\sqrt{\frac{\pi}{2}} .
$$

4. Substitute all values of $\alpha_{i}, i=0, \ldots, 4$ into (25), we have

$$
\nu_{0}=\sum_{k=0}^{4} \alpha_{k} \phi_{k}(x)=\sqrt{\frac{\pi}{2}} \phi_{3}(x)=8 x^{3}+4 x^{3}-4 x-1 .
$$

Thus, we obtain the approximate solution in the form

$$
\varphi(x)=\sqrt{1-x^{2}}\left(8 x^{3}+4 x^{3}-4 x-1\right)
$$

which is same as exact solution. Modified HPM has zero error for solving Eq. (62).

Example 5 Let us rewrite Eq. (4) in the form of

$$
\frac{1}{\pi} f_{-1}^{1} \frac{2+t x(t-x)}{(x-t)^{2}} \varphi(t) d t+\frac{1}{\pi} \int_{-1}^{1}\left(\frac{1}{t+2}+\frac{1}{x+2}\right) \varphi(t) d t=f(x), \quad-1<x<1,
$$

where $f(x)=-\frac{20 \sqrt{3}}{2+x^{2}}-\frac{10 x^{2}}{x+2}(2-\sqrt{3}+x)+10(2-\sqrt{3}) x+\frac{10}{3}(2 \sqrt{3}-3)+\frac{10(2-\sqrt{3})}{x+2}$.

The exact solution of Eq. $(70)$ is $\varphi(x)=\sqrt{1-x^{2}} \frac{10}{x+2}$.

Solution Standard HPM is not suitable for solving the Eq. (70) as it is not satisfies the conditions in Theorem 8. For the modified HPM, we choose the selective functions $g_{j}(x)=\phi_{j}(x), j=0, \ldots, m$. Approximating $L(x, t)$ in Chebyshev polynomials form as follows

$$
L_{n}(x, t)=x+\frac{1}{x+2}+\sum_{k=0}^{n}(-1)^{k} \frac{t^{k}}{2^{k+1}} .
$$


In this case $\tilde{L} \neq 0$, therefore the scheme (30) has the form

$$
\begin{aligned}
& \left(H+C+L_{n}\right) v_{0}=\sum_{j=0}^{m} \alpha_{j} \phi_{j}(x), \\
& \left(H+C+L_{n}\right) v_{1}=-\tilde{L}+f-\sum_{j=0}^{m} \alpha_{j} \phi_{j}(x) .
\end{aligned}
$$

To solve Eq. (70), we choose the collocation points, $x_{i}$ as the roots of $\phi(x)$ which is

$$
x_{i}=\cos \frac{(i+1) \pi}{n+2}, \quad i=0,1, \ldots n
$$

Errors of $\varphi(x)$ using modified HPM for values of $m=\{6,26\}$ are presented in Table 5 .

From Tables 1, 2 and 4 show the comparison between the past method with HPM and modified HPM. It is clearly seen that Modified HPM gives more accurate results compare to the Chebyshev expansion method Mahiub et al. (2011), Bernstein polynomials approach Mandal and Bhattacharya (2007) and Reproducing Kernel method Chen and Zhou (2011).

Table 5 conclude that the modified HPM converges to the exact solution of Eq. (70) by increasing the number of collocation points $n$ and number of selection functions $m$. It can also be seen that the convergence is achieved at all singular points $x$ including the one which is close to the end points of the interval $[-1,1]$.

\section{Conclusion}

In this work, the standard and modified HPM are used to find the approximate solution of the first kind HSIE. The theoretical aspect supported by the same numerical examples have shown the modified HPM gives better approximation than the standard HPM. Based on the examples, the modified HPM ables to handle the problem that can not be solved by standard HPM. Modified HPM is effective and reliable method for solving HSIE of the first kind of the form (4).

Table 5 Errors of solution for Eq. (70) solved by modified HPM

\begin{tabular}{lll}
\hline $\boldsymbol{x}$ & Modified HPM, $\boldsymbol{m}=\boldsymbol{n}=\mathbf{6}$ & Modified HPM, $\boldsymbol{m}=\boldsymbol{n}=\mathbf{6}$ \\
\hline-0.9999 & $1.0851729 \times 10^{-4}$ & $1.4040446 \times 10^{-10}$ \\
-0.901 & $3.5501594 \times 10^{-4}$ & $1.8203460 \times 10^{-9}$ \\
-0.725 & $1.8899796 \times 10^{-4}$ & $6.1943369 \times 10^{-8}$ \\
-0.436 & $3.0648319 \times 10^{-4}$ & $2.6221447 \times 10^{-8}$ \\
-0.015 & $8.6784464 \times 10^{-5}$ & $1.7385004 \times 10^{-8}$ \\
0.015 & $1.9992451 \times 10^{-5}$ & $1.8524990 \times 10^{-8}$ \\
0.436 & $3.3916634 \times 10^{-1}$ & $1.9700974 \times 10^{-8}$ \\
0.725 & $6.3326189 \times 10^{-5}$ & $2.3479494 \times 10^{-8}$ \\
0.901 & $1.2745747 \times 10^{-4}$ & $1.3403888 \times 10^{-8}$ \\
0.9999 & $3.3327100 \times 10^{-5}$ & $3.5400390 \times 10^{-10}$
\end{tabular}




\section{Authors' contributions}

ZKE and ZM carried out mainly theoretical investigations (Section 2 and 4) of the HPM and modified HPM for Hypersingular integral equations and norm convergence for both HPM and modified HPM are proved. NMANL carried out mainly in Introduction and literature review which is Section 1. FSZ participated in the derivation of HPM and modified HPM together with numerical results which is Section 3 and 5, moreover she helped to draft the manuscript. All authors read and approved the final manuscript.

\section{Author details}

${ }^{1}$ Faculty of Science and Technology, Universiti Sains Islam Malaysia (USIM), Negeri Sembilan, Malaysia. ${ }^{2}$ Department of Mathematics, Faculty of Science, Universiti Putra Malaysia (UPM), Selangor, Malaysia. ${ }^{3}$ Institute for Mathematical Research, Universiti Putra Malaysia (UPM), Selangor, Malaysia. ${ }^{4}$ Faculty of Mathematics and Mechanics, Samarkand State University, Samarkand, Uzbekistan.

\section{Acknowledgements}

This work was supported by University Putra Malaysia (UPM) and Universiti Sains Islam Malaysia (USIM) under Research Grands (Research Grand of UPM, project code is GP-i(2014) 9442300 and Research Grand of USIM PPP/GP/FST/30/14915). Authors are grateful for sponsor and financial support of the Research Management Center (RMC) of UPM and USIM.

\section{Competing interests}

The authors declare that they have no competing interests.

Received: 23 April 2016 Accepted: 12 August 2016

Published online: 01 September 2016

\section{References}

Berthold D, Hoppe W, Silbermann B (1992) A fast algorithm for solving the generalized airfoil equation. J Comput Appl Math 43(12):185-219

Boykov IV, Ventsel ES, Boykova Al (2010) An approximate solution of hypersingular integral equations. Appl Number Math 60:607-628

Boykov IV, Ventsel ES, Roudnev VA, Boykova Al (2014) An approximate solution of nonlinear hypersingular integral equations. Appl Number Math 86:1-21

Chan YS, Fannjiang AC, Paulino GH (2003) Integral equations with hypersingular kernels theory and applications to fracture mechanics. Internat J Eng Sci 41:683-720

Chen Z, Zhou YF (2011) A new method for solving hypersingular integral equations of the first kind. Appl Math Lett 24:636-641

Dehghan M, Shakeri F (2008) Solution of an integro-differential equation arising in oscillating magnetic fields using He's homotopy perturbation method. Prog Electromagn Res 78:361-376

Eshkuvatov ZK, Nik Long NMA, Abdulkawi M (2009) Approximate solution of singular integral equations of the first kind with Cauchy kernel. Appl Math Lett 22(5):651-657

Ghasemi M, Kajani MT, Davari A (2007) Numerical solution of the nonlinear Volterra-Fredholm integral equations by using homotopy perturbation method. Appl Math Comput 188:446-449

Ghorbani A, Saberi-Nadjafi J (2006) Exact solutions for nonlinear integral equations by a modified homotopy perturbation method. Comput Math Appl 28:1032-1039

Golbabai A, Javidi M (2007) Application of He's homotopy perturbation method for nth-order integro-differential equations. Appl Math Comput 190:1409-1416

Golberg MA (1987) The convergence of several algorithms for solving integral equations with finite part integrals. II. Appl Math Comput 21:283-293

He JH (1999) Homotopy perturbation technique. Comput Methods Appl Mech Eng 178:257-262

He JH (2000) A coupling method of a homotopy technique and a perturbation technique for non-linear problems. Int J Nonlinear Mech 35:37-43

Jafari H, Alipour M, Tajadodi H (2010) Convergence of homotopy perturbation method for solving integral equations. Thai J Math 8:511-520

Javidi M, Golbabai A (2009) Modified homotopy perturbation method for solving non-linear Fredholm integral equations. Chaos Solitons Fractals 40:1408-1412

Kanoria M, Mandal BN (2002) Water wave scattering by a submerged circular-arc-shaped plate. Fluid Dyn 31:317-331

Khan Y, Wu Q (2011) Homotopy perturbation transform method for nonlinear equations using He's polynomials. Comput Math Appl 61:1963-1967

Madani M, Fathizadeh M, Khan Y, Yildrim A (2011) On coupling of the homotopy perturbation method and Laplace transformation. Math Comput Model 53:1937-1945

Mahiub Mohammad Abdulkawi, Nik Long NMA, Eshkuvatov ZK (2011) Numerical solution of hypersingular integral equations. Int J Pure Appl Math 69(3):265-274

Mandal BN, Bhattacharya Subhra (2007) Numerical solution of some classes of integral equations using Bernstein polynomials. Appl Math Comput 190:1707-1716

Mohamad Nor H, Md Ismail Al, Abdul Majid A (2013) A new homotopy function for solving nonlinear equations. AIPCP 1557:21-25

Nik Long NMA, Eshkuvatov ZK (2009) Hypersingular integral equation for multiple curved cracks problem in plane elasticity. Internat J Sol Struct 46:2611-2617 
Okayama T, Matsuo T, Sugihara M (2011) Improvement of a Sinc-collocation method for Fredholm integral equations of the second kind. BIT Numer Math 51:339-366

Panda Srikumar (2013) Assessment of homotopy analysis method and modified homotopy perturbation method for strongly nonlinear oscillator. Int J Nonlinear Sci 16(4):291-300

Panda S, Martha SC, Chakrabarti A (2015) A modified approach to numerical solution of Fredholm integral equations of the second kind. Appl Math Comput 271:102-112

Parsons NF, Martin PA (1994) Scattering of water waves by submerged curved plates and by surface-piercing flat plates. Appl Ocean Res 16:129-139

Ramos II (2008) Piecewise homotopy methods for nonlinear ordinary differential equations. Appl Math Comput 198:92-116

Reed M, Simon B (1980) Functional analysis, vol 1. Methods of modern mathematical physics. Academic Press, New York Słota D (2010) The application of the homotopy perturbation method to one-phase inverse Stefan problem. Int Commun Heat Mass Trans 37:587-592

\section{Submit your manuscript to a SpringerOpen ${ }^{\circ}$} journal and benefit from:

- Convenient online submission

\section{- Rigorous peer review}

- Immediate publication on acceptance

- Open access: articles freely available online

- High visibility within the field

- Retaining the copyright to your article 